\title{
Significant bradycardia in patient with COVID-19 sepsis: Full management review
}

\author{
Lac Nguyen ${ }^{* 1}$, Chuong Le ${ }^{1}$, Steven Pham ${ }^{2}$, San Tran ${ }^{3}$, Thanh Hoang ${ }^{4}$, Khiet Nguyen ${ }^{1}$, Thoai Nguyen ${ }^{1}$, Hashim \\ Chaudhry ${ }^{5}$, Nghi Tran ${ }^{1}$, Ngoc Mai ${ }^{6}$, Michael Brockman ${ }^{5}$, Vien Phan ${ }^{7}$, Amandeep Goyal ${ }^{8}$, Phillip Tran ${ }^{9}$ \\ ${ }^{1}$ University of Medicine and Pharmacy at Ho Chi Minh city, Vietnam \\ ${ }^{2}$ Kansas City University College of Osteopathic Medicine, Missouri, USA \\ ${ }^{3}$ Mount Sinai Hospital Medical Center of Chicago, USA \\ ${ }^{4}$ Faculty of Medicine, Vietnam Military Medical University, Vietnam \\ ${ }^{5}$ AT Still School of Osteopathic Medicine, Arizona, USA \\ ${ }^{6}$ Pham Ngoc Thach University of Medicine, Vietnam \\ ${ }^{7}$ First Department of Internal Medicine, Kansai Medical University, Osaka, Japan \\ ${ }^{8}$ University of Kansas Medical Center, Kansas, USA \\ ${ }^{9}$ Nam Can Tho University Medical Center, Vietnam
}

Received: July 14, 2021

DOI: $10.5430 /$ crim.v8n1p17
Accepted: August 30, 2021

Online Published: September 15, 2021

\begin{abstract}
Cardiovascular manifestations of COVID-19 infection remain an ongoing study. We present a case of significant bradycardia in the patient with COVID-19 sepsis. We discuss about the possible mechanism, pathophysiology, and management in this pathology.
\end{abstract}

Key Words: COVID-19, Bradycardia, Sepsis

\section{INTRODUCTION}

Sepsis, a condition where organs are damaged due to a dysregulated response to an infection, is one of the leading causes of death across United States hospitals. In fact, one out of three deaths in the hospital come from sepsis. ${ }^{[1]}$ Most patients with sepsis present with tachycardia. The opposite, low heart rate, a condition called "bradycardia," is rarely described. We present a case of patient who presented with COVID-19 pneumonia which progressed to sepsis, and later found to be bradycardic.

\section{Case presentation}

An 80-year-old man with history of hypertension, hyperlipidemia, and benign prostatic hyperplasia was hospitalized in December 2020 for COVID-19 pneumonia. He was treated with Remdesivir and steroids. He was then discharged. Unfortunately, a week later one, he was found to have sepsis with leukocytosis (white blood cell of 43.6). Patient also developed acute kidney injury (AKI) with Creatinine 4.1 (his baseline is 0.8 ), and transient hypotension (blood pressure 75/58), requiring Norepinephrine drip. At second admission on 12/26/2020, patient had a heart rate (HR) of 101 (see Figure 1). Two days after, patient developed constant sinus

\footnotetext{
*Correspondence: Lac Nguyen; Email: nglachanyds@gmail.com; Address:University of Medicine and Pharmacy at Ho Chi Minh City, 217 Hong Bang, Ward 11, District 05, Ho Chi Minh City, Vietnam.
} 
bradycardia with HR in 30s-50s range for four days (see gram (EKG) and elevated Troponin at 0.37. Echocardiogram Figure 2). The lowest HR was 36 while awake (see Figure 3). 01/02/2021 was overall unremarkable with left ventricular Urine culture was positive for two bacteria, which was possi- ejection fraction (LVEF) 60\%-65\%, grade 1 diastolic dysbly contaminated. Blood culture was negative for four bottles function, and right ventricular systolic pressure (RVSP) of in two consecutive days. Patient denies chest pain and syn- $26 \mathrm{mmHg}$. cope. There were non-specific ST changes on electrocardio-

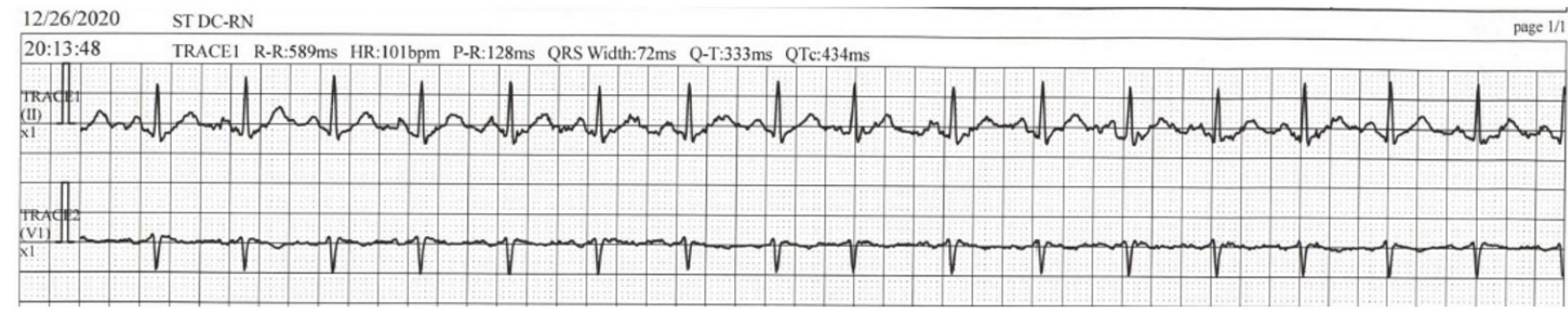

Figure 1. Sinus tachycardia on 12/26/2020 Day 1 on second admission (HR 101)

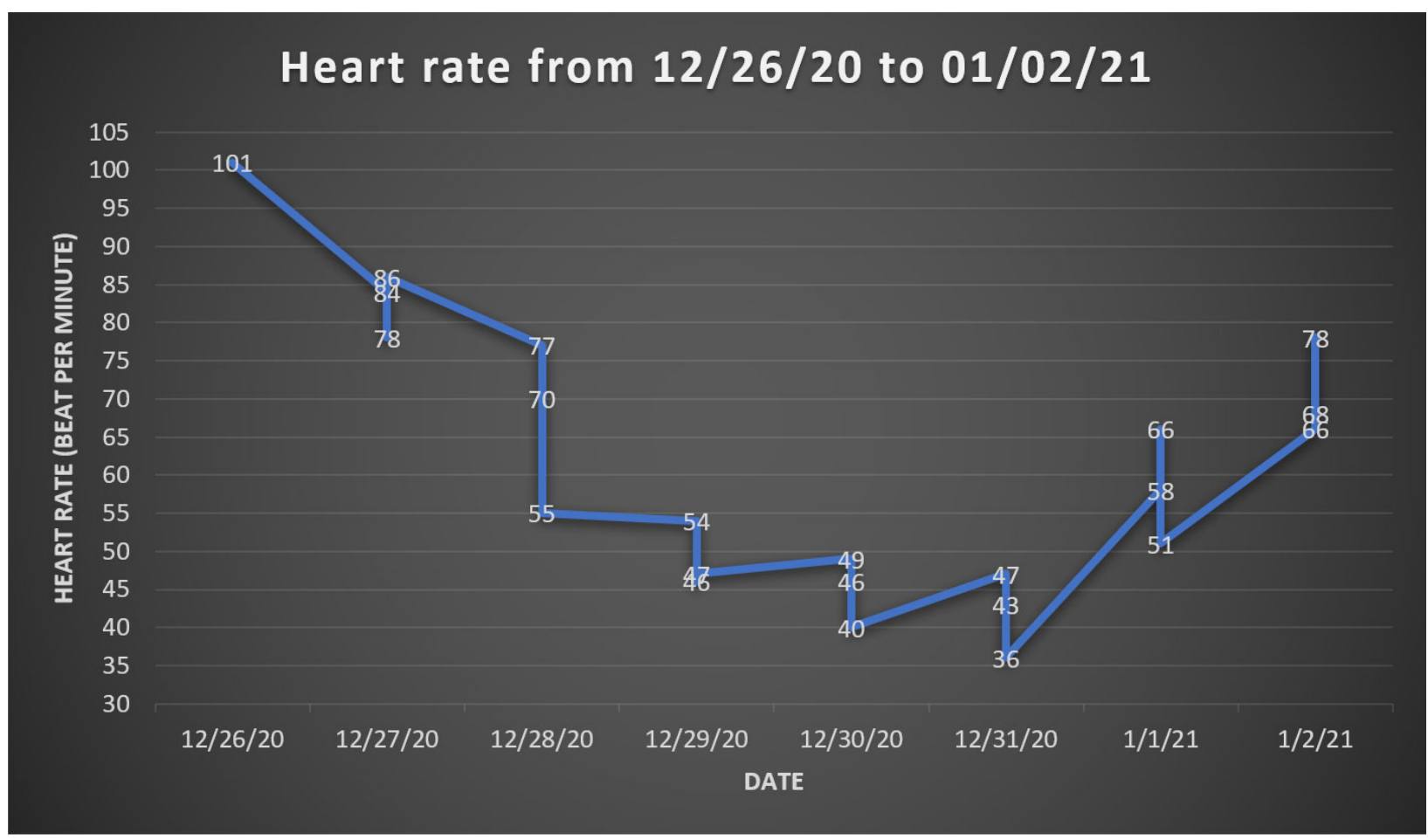

Figure 2. Linear graph showing heart rate of patient from tachycardia (max HR 101) to bradycardia (min HR 36) during sepsis phase

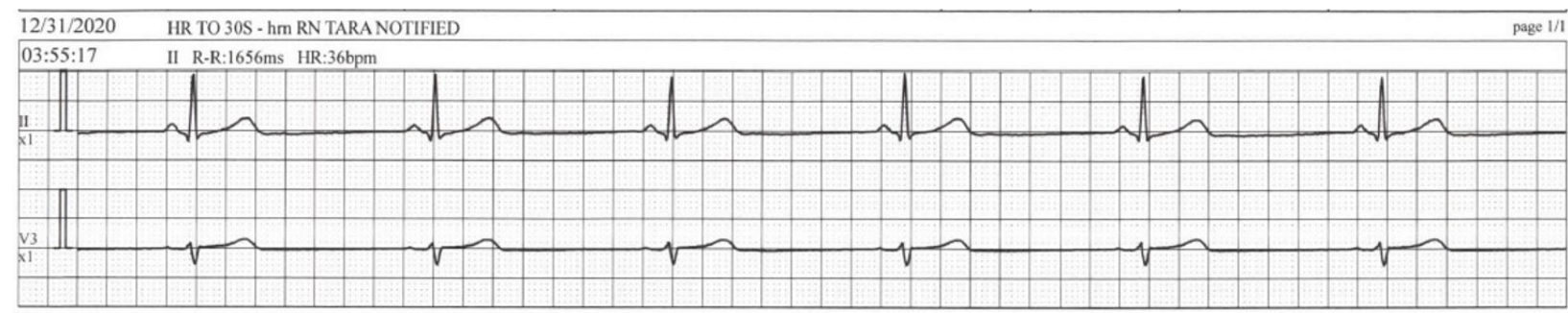

Figure 3. Sinus bradycardia on 12/31/2020 during sepsis (HR 36) 
Cardiac telemetry monitor at home for seven days showed sinus rhythm with an average HR of 76 (HR ranging from 58123). His recovered HR was 78 (see Figure 4). There were rare premature ventricular contractions (PVCs) and premature atrial contractions (PACs). Nuclear stress test revealed no evidence of ischemia.

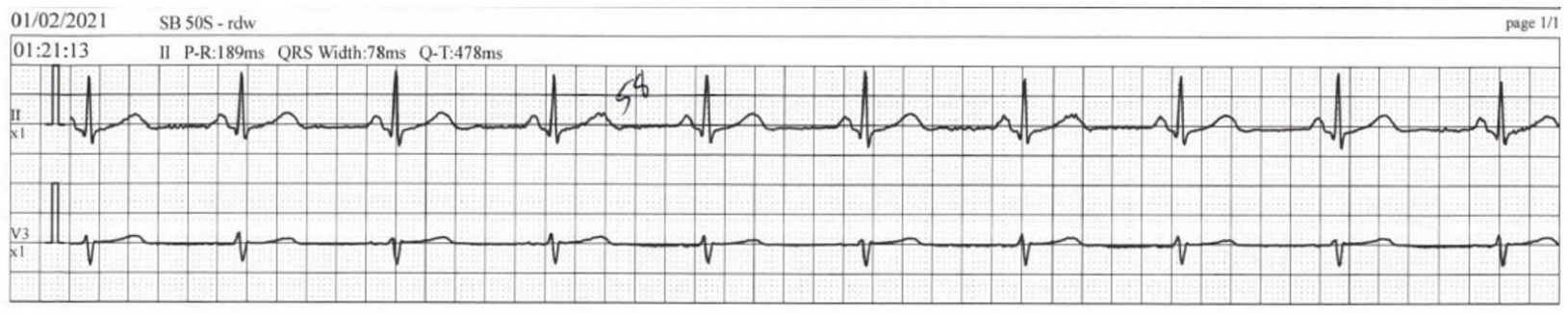

Figure 4. Recovery of HR (HR 78)

On follow-up on April 2021, patient was asymptomatic. His physical examination was unremarkable, and EKG demonstrated normal sinus rhythm.

\section{DisCuSSION}

Since December 2019, the world has been suffering from devastating pandemic caused by a novel virus SARS-CoV-2. By January 2021, the number of people who were infected with COVID-19 have surpassed 80 million worldwide, leading to over 1.7 million deaths. In addition to respiratory manifestations, various cardiac complications have been increasingly reported in patients with COVID-19 infection, including but not limited to acute coronary syndrome, heart failure, left ventricular dysfunction, and myocarditis. ${ }^{[2]}$ Arrhythmias and correlated symptoms have also been reported, with sinus tachycardia being the most common finding. However, bradycardia is relatively uncommon $(0.9 \%-30.8 \%) .^{[2-7]}$ The time of onset of bradycardia varies from 4 days to 3 weeks. ${ }^{[2,7]}$ During bradycardic episodes, patients usually had normal cardiac biomarkers, normal EKG, and echocardiogram. ${ }^{[8-12]}$ Fortunately, most of symptoms did not persist after patients recovered from COVID-19 infection. ${ }^{[6]}$

\subsection{Mechanism}

The underlying etiology of bradycardia in COVID-19 patients seems to be multifactorial. ${ }^{[2,13]}$ The proposed mechanisms include respiratory dysfunction resulting in hypoxia, systemic and local inflammatory responses releasing proinflammatory cytokines, myocardial injury, and side effects of drugs. The normal cardiac biomarkers values commonly found in COVID-19 patients experiencing bradycardia favor the hypothesis that the virus causes bradycardia through other pathways rather than direct cardiac cell destruction. ${ }^{[6,8,10,12,14]}$ The arrhythmogenicity of SARS-CoV2 remains controversial.

This new coronavirus has been well-documented to invade cells using angiotensin-converting enzyme 2 (ACE-2) recep-

Published by Sciedu Press tor, which is widely expressed in the cardiovascular system including cardiomyocytes, endothelial cells, macrophages, and other interstitial cells. The presence of ACE-2 in SA node reported in recent animal studies and the conduction disturbances following altered ACE- 2 expression suggested possible direct inhibitory effect of SARS-CoV-2 on SA node and conduction system. ${ }^{[6,8]}$

Another hypothesis suggested that the coronavirus may cause dysfunction of the cardiorespiratory center along with autonomic nervous system. Several cases have been reported to be able to spread from the respiratory airways to the medullary cardiorespiratory center through synaptic transmission. This hypothesis may take responsibility for the simultaneously presence of both respiratory and cardiac collapse in numerous COVID-19 infected patients. ${ }^{[15]}$ In addition, altered ion channel functions secondary to interstitial edema, abnormal intercellular coupling and another features of local inflammatory process could also be involved, as seen in other arrythmias in viral infection. ${ }^{[16]}$

On the other hand, the elevation of inflammatory markers may play a major role in eliciting inflammatory response in the etiology of bradycardia. The pro-inflammatory cytokines, most prominently IL-6, significantly contribute to the pathogenesis of relative bradycardia. The excessive release of cytokines is suggested as a sign of worsening inflammatory reactions as well as a prediction of upcoming cytokine storm. ${ }^{[7]}$ Additionally, the currently used medications for patients with COVID-19 infections may also contribute to the bradycardia in such patients. Anti-infective medications (azithromycin, chloroquine, lopinavir/ritonavir/remdesivir) or supportive treatment (propofol) potentially cause arrhythmia and bradycardia. ${ }^{[2,7,9,13]}$

\subsection{Relative bradycardia}

Relative bradycardia describes the phenomenon where the heart rate does not increase appropriately with an increase 
of body temperature. Even though this phenomenon is described in multiple infectious and non-infectious conditions, the pathogenesis still remains poorly understood. ${ }^{[17]}$ Proposed mechanisms include direct effects of pathogen on the SA node as well as involvement of inflammatory cytokines. IL-6, a cytokine that plays a significant role in the "cytokine storm" commonly found in COVID-19 patients, appears to correlate the most with low heart rate variability. ${ }^{[18,19]}$ As a result, relative bradycardia is suggested as a clinical feature of COVID-19. This characteristic may become useful to distinguish community-acquired pneumonia caused by COVID-19 or bacteria. ${ }^{[18,20-22]}$

\subsection{Management/ Treatment}

To date, as our acknowledgement of bradycardia caused by SARS-CoV-2 remains in its infancy, the specific treatment has not been well-established. The current management plan mainly focuses on treating the underlying COVID-19 infection and possible correlated symptoms following relevant guidelines. Transient bradycardia can be resolved spontaneously by the recovery from COVID-19 infection without any additional interventions. ${ }^{[7,16]}$ Isoproterenol could be given intravenously to help stabilize vital signs, deferring the use of pacemaker devices. ${ }^{[6,23]}$ In some cases, pacing therapy with either pacemaker pads or permanent pacemaker may be necessary. ${ }^{[8,10,14,15,23]}$ Leadless pacemaker was proposed in patients requiring permanent pacemaker to lower the risk of virus transmission to healthcare workers as well as reducing cardiac and IV infections. ${ }^{[14]}$ Based on the proposed mechanisms, anti-inflammatory medications have been suggested as possible therapeutic agents for cardiovascular complications in COVID-19 patients. ${ }^{[16]}$

\subsection{Prognosis/ Follow-up}

In most cases, rhythmic abnormalities resolved within less than 2 weeks or even less than 24 hours. ${ }^{[7,8,16]}$ However, the symptoms may persist in some patients despite recovery from COVID-19 infections. ${ }^{[9,12,23]}$ The prognostic value of bradycardia in COVID-19 patients remains unclear. Patients with severe bradycardia during COVID infection could have high short-term mortality and morbidity. ${ }^{[14]}$ However, the accuracy of this result may be questionable due to the small sample size (7 patients) as well as the difference in severity of COVID-19 infection and comorbidities in those patients. This problem should be established in further studies with larger sample size. Most of aforementioned studies proposed frequent vital signs, inflammatory markers monitoring, and ECG reevaluation on subsequent follow-up.

\section{CONFLICTS OF INTEREST DISCLOSURE}

The authors have declared no conflicts of interest.

\section{REFERENCES}

[1] Beltrán-García J, Osca-Verdegal R, Pallardó FV, et al. Sepsis and Coronavirus Disease 2019: Common Features and AntiInflammatory Therapeutic Approaches. Crit Care Med. 2020; 48(12): 1841-1844. PMid:32826431. https://doi.org/10.1097/CCM. 0000000000004625

[2] Kochi AN, Tagliari AP, Forleo GB, et al. Cardiac and arrhythmic complications in patients with COVID-19. J Cardiovasc Electrophysiol. 2020;31(5):1003-8. PMid:32270559. https ://doi . org/10.1 $111 /$ jce. 14479

[3] Yu CM, Wong RS, Wu EB, et al. Cardiovascular complications of severe acute respiratory syndrome. Postgrad Med J. 2006; 82(964): 140-4. PMid:16461478. https://doi .org/10.1136/pgmj . 200 5.037515

[4] Kunal S, Sharma SM, Sharma SK, et al. Cardiovascular complications and its impact on outcomes in COVID-19. Indian Heart J. 2020; 72(6): 593-8. PMid:33357651. https://doi .org/10.1016/j. ih j.2020.10.005

[5] Chen Q, Xu L, Dai Y, et al. Cardiovascular manifestations in severe and critical patients with COVID-19. Clin Cardiol. 2020; 43(7): 796802. PMid:32562427. https://doi.org/10.1002/clc.23384

[6] Hu L, Gong L, Jiang Z, et al. Clinical analysis of sinus bradycardia in patients with severe COVID-19 pneumonia. Crit Care. 2020; 24(1): 257. PMid:32456695. https://doi .org/10.1186/s13054-020 $-02933-3$
[7] Amaratunga EA, Corwin DS, Moran L, et al. Bradycardia in Patients With COVID-19: A Calm Before the Storm? Cureus. 2020; 12(6): e8599. PMid:32550090. https://doi.org/10.7759/cureus.8 599

[8] Kir D, Mohan C, Sancassani R. Heart Brake: An Unusual Cardiac Manifestation of COVID-19. JACC Case Rep. 2020; 2(9): 1252 5. PMid:32368756. https://doi .org/10.1016/j.jaccas . 202 0.04 .026

[9] Peigh G, Leya MV, Baman JR, et al. Novel coronavirus 19 (COVID19) associated sinus node dysfunction: a case series. Eur Heart J Case Rep. 2020; 4(FI1): 1-6. PMid:33089039. https ://doi .org/10.1 093/ehjcr/ytaa132

[10] Eid MM. COVID-19 patient with symptomatic bradycardia. Vis J Emerg Med. 2021; 22: 100920. PMid:33200102. https ://doi .or g/10.1016/j.visj.2020.100920

[11] Azarkish M, Laleh Far V, Eslami M, et al. Transient complete heart block in a patient with critical COVID-19. Eur Heart J. 2020; 41(22): 2131. PMid:32285920. https://doi.org/10.1093/eu rheartj/ehaa307

[12] Haddadin FI, Mahdawi TE, Hattar L, et al. A case of complete heart block in a COVID-19 infected patient. J Cardiol Cases. 2021; 23(1): 27-30. PMid:32904735. https://doi.org/10.1016/j.jccase .2020 .08 .006

[13] Basu-Ray I, Almaddah N, Adeboye A, et al. Cardiac Manifestations Of Coronavirus (COVID-19). StatPearls. Treasure Island (FL)2021. 
[14] Chinitz JS, Goyal R, Harding M, et al. Bradyarrhythmias in patients with COVID-19: Marker of poor prognosis? Pacing Clin Electrophysiol. 2020; 43(10): 1199-204. PMid:32820823. https : //doi.org/10.1111/pace. 14042

[15] Cimino G, Pascariello G, Bernardi N, et al. Sinus Node Dysfunction in a Young Patient With COVID-19. JACC Case Rep. 2020; 2(9): 1240-4. PMid:32835265. https://doi.org/10.1016/j.jaccas .2020 .05 .067

[16] Babapoor-Farrokhran S, Batnyam U, Wiener PC, et al. Atrioventricular and Sinus Node Dysfunction in Stable COVID-19 Patients. SN Compr Clin Med. 2020: 1-4. PMid:32901230. https ://doi.org/ 10.1007/s42399-020-00497-5

[17] Cunha BA. The diagnostic significance of relative bradycardia in infectious disease. Clin Microbiol Infect. 2000; 6(12): 633-4. PMid:11284920.https://doi.org/10.1046/j.1469-0691. 20 $00.0194 f . x$

[18] Capoferri G, Osthoff M, Egli A, et al. Relative bradycardia in patients with COVID-19. Clin Microbiol Infect. 2021; 27(2): 295-6. PMid:32822885. https ://doi.org/10.1016/j.cmi.2020.08. 013
[19] Ye F, Winchester D, Stalvey C, et al. Proposed mechanisms of relative bradycardia. Med Hypotheses. 2018; 119: 63-7. PMid:30122494. https://doi.org/10.1016/j.mehy.2018.07.014

[20] Ikeuchi K, Saito M, Yamamoto S, et al. Relative Bradycardia in Patients with Mild-to-Moderate Coronavirus Disease, Japan. Emerg Infect Dis. 2020; 26(10): 2504-6. PMid:32610036. https ://doi . org/10.3201/eid2610.202648

[21] Nakamura K, Ide S, Saito S, et al. COVID-19 can suddenly become severe: a case series from Tokyo, Japan. Glob Health Med. 2020; 2(3): 174-7. PMid:33330803. https://doi.org/10.35772/ghm .2020 .01054

[22] Hiraiwa H, Goto Y, Nakamura G, et al. Relative bradycardia as a clinical feature in patients with coronavirus disease 2019 (COVID19): A report of two cases. J Cardiol Cases. 2020; 22(6): 260-4. PMid:32837666. https://doi.org/10.1016/j.jccase. 2020. 07.015

[23] Ashok V, Loke WI. Case report: high-grade atrioventricular block in suspected COVID-19 myocarditis. Eur Heart J Case Rep. 2020; 4(FI1): 1-6. PMid:33089060. https ://doi.org/10.1093/ehjc r/ytaa248 